\title{
IRIDOSQUISIS ASOCIADA A SÍFILIS CONGÉNITA: CONFIRMACIÓN SEROLÓGICA A LOS 80
}

\author{
IRIDOSCHISIS ASSOCIATED TO CONGENITAL SYPHILIS: \\ SEROLOGICAL CONFIRMATION AT THE 80'S
}

\author{
PÉREZ-CARRO G ${ }^{1}$, VILANOVA Mㄹ ANTUÑA MG ${ }^{2}$, CÁRCABA V ${ }^{3}$, JUNCEDA-MORENO J
}

\begin{abstract}
RESUMEN
Caso clínico: Mujer octogenaria que acude por dolor ocular unilateral. En la exploración observamos, además de una abrasión corneal, una iridosquisis bilateral con un iris desflecado, desdoblado entre sus capas estromales.

Discusión: Esta rara entidad está relacionada con los cambios degenerativos seniles o al glaucoma de ángulo cerrado. Sin embargo, también es asociado a la sífilis congénita con o sin la coexistencia de queratitis intersticial. Las conjuntivitis en la infancia y el fondo de ojo en «sal y pimienta» complementan el diagnóstico de sospecha. Confirmándose, a los 80 años una sífilis congénita tardía curada, por los test serológicos treponémicos (RPR-) y no treponémicos (TPHA+).
\end{abstract}

Palabras clave: Iridosquisis bilateral, glaucoma ángulo cerrado, sífilis congénita, queratitis intersticial.

\begin{abstract}
Clinical case: Female in her eighties is admitted suffering from unilateral ocular pain. On examination we observed, as well as corneal abrasion, a bilateral iridoschisis with a frayed iris, unfolded between its stromal layers.

Discussion: This rare case is related either to senile degenerative change or to angle-closure glaucoma. However, it is also associated with congenital syphilis with or without the presence of interstitial keratitis. Infant conjunctivitis and a «salt and pepper» appearance of the fundus oculi complete the diagnosis.

It is confirmed that the patient had suffered from congenital late syphilis, cured by the age of 80: this confirmation is reached by treponemal (RPR-) and non-treponemal (TPHA+) serological tests (Arch Soc Esp Oftalmol 2009; 84: 353-358).
\end{abstract}

Key words: Bilateral iridoschisis, angle-closure glaucoma, congenital syphilis, interstitial keratitis.

\footnotetext{
Recibido: 20/5/08. Aceptado: 5/7/09.

Hospital del Valle del Nalón. Asturias. España.

${ }^{1}$ Licenciada en Medicina. Servicio de Oftalmología.

2 Licenciada en Farmacia. Servicio de Análisis Clínicos.

3 Doctor en Medicina. Servicio de Medicina Interna.

${ }^{4}$ Doctor en Medicina. Servicio de Oftalmología.

Presentado como comunicación en formato digital en la 21 Reunión Nacional del GEMU 2008.

Correspondencia:

Gema Pérez Carro

C/. Velázquez, $2,2 .^{\circ}$ I

33211 Gijón (Asturias)

España

E-mail: gemurri27@yahoo.es
} 


\section{INTRODUCCIÓN}

La iridosquisis (IQ) es una rara alteración, manifestación asociada a cambios atróficos seniles o a la coexistencia de glaucoma de ángulo estrecho. Sin embargo encontramos su asociación a sífilis congénita con o sin queratitis intersticial (QI). Se trata de un desdoblamiento del estroma del iris que va desde la atrofia simple a un desgarro extenso de la cara anterior del iris. Presentamos un caso de iridosquisis, hallado de forma casual y su asociación a la sífilis congénita, su diagnóstico orientado por la historia clínica y confirmada por test de laboratorio.

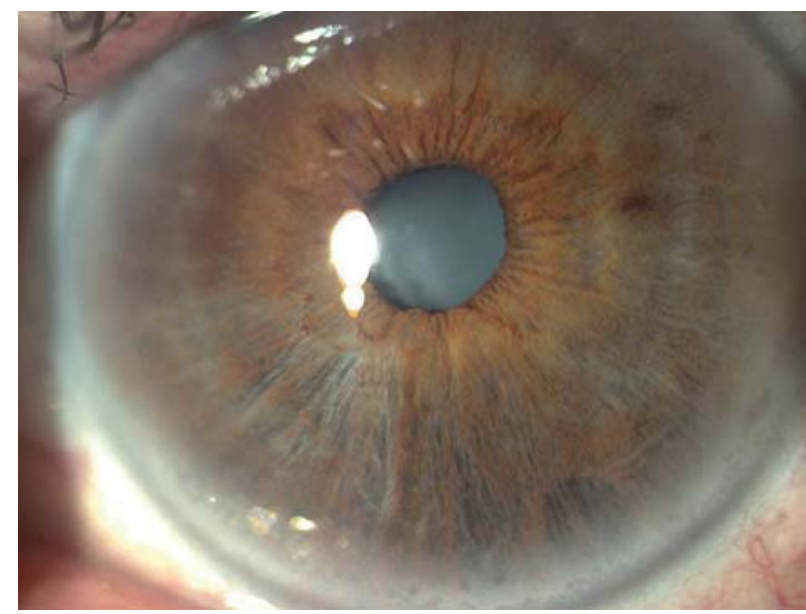

Fig. 1a: Iridosquisis OI más acusada, mayor atrofia del iris.

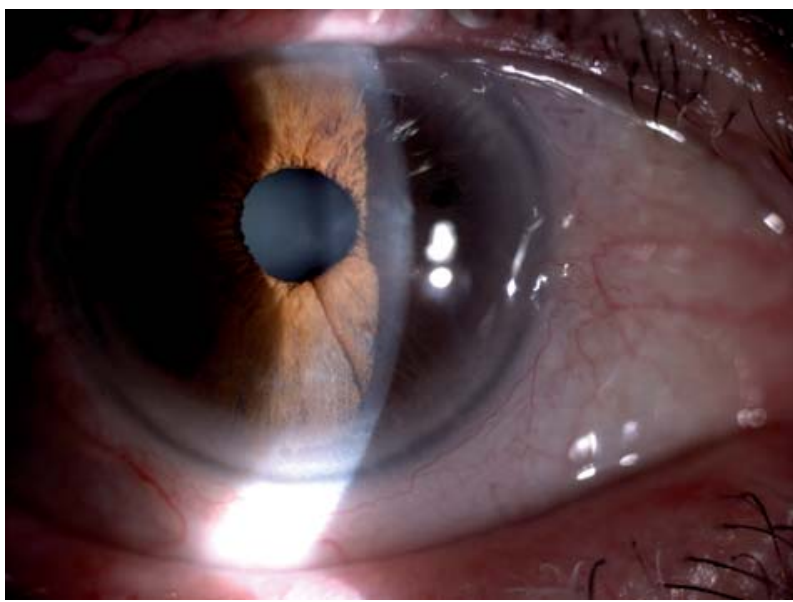

Fig. 1b: Iridosquisis OI donde se aprecia desdoblamiento del iris, globuloso.

\section{CASO CLÍNICO}

Mujer de 80 años, no conocida del servicio, acude por presentar dolor traumático en su ojo izquierdo (OI). Entre sus antecedentes destacamos conjuntivitis de repetición en la infancia, artrosis senil y 4 hijos sanos. En la exploración oftalmológica observamos una abrasión corneal en OI, que curará con oclusión y pomada epitelizante en 1 semana. Además se aprecia en ambos ojos (AO) un iris atrófico, desdoblado (figs. 1a y 1b), desflecado con atrofia en sector inferior, más en OI (figs. 1c, 1d, 1e, 1f y 1g) y una reducción de la cámara anterior visible también en la gonioscopia (figs. 2 a y 2b). Presenta

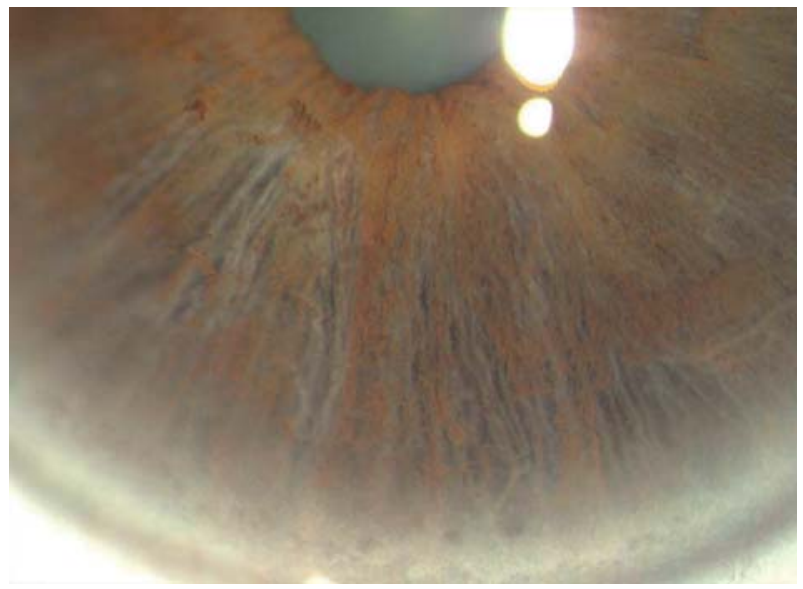

Fig. 1c: Atrofia parcheada del iris OI.

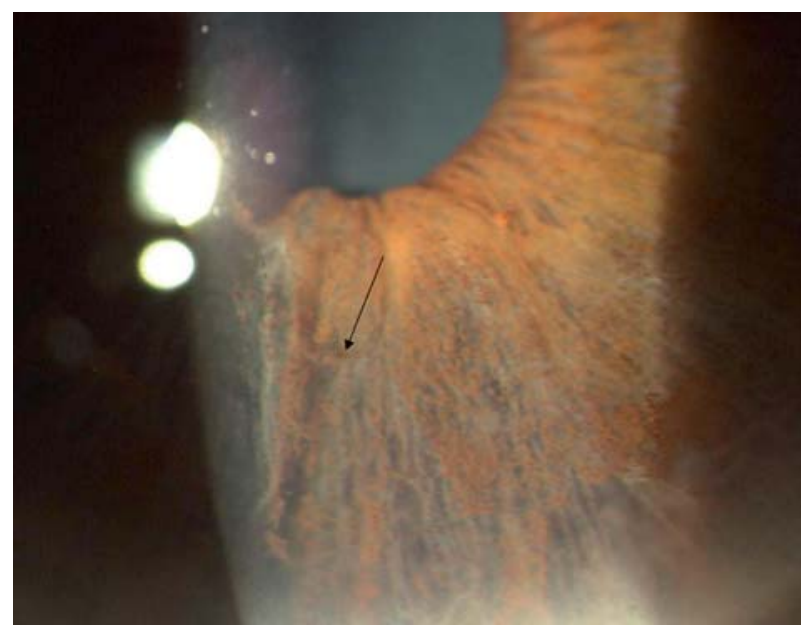

Fig. 1d: Fibrillas del iris. 


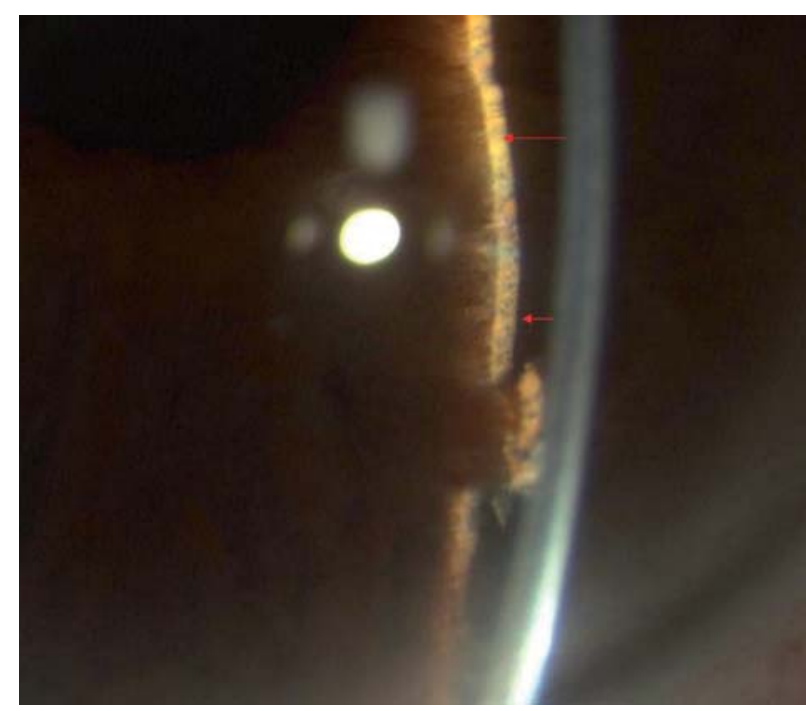

Fig. 1e: Estrechamiento de la cámara anterior OI, protrusión de material iridiano.

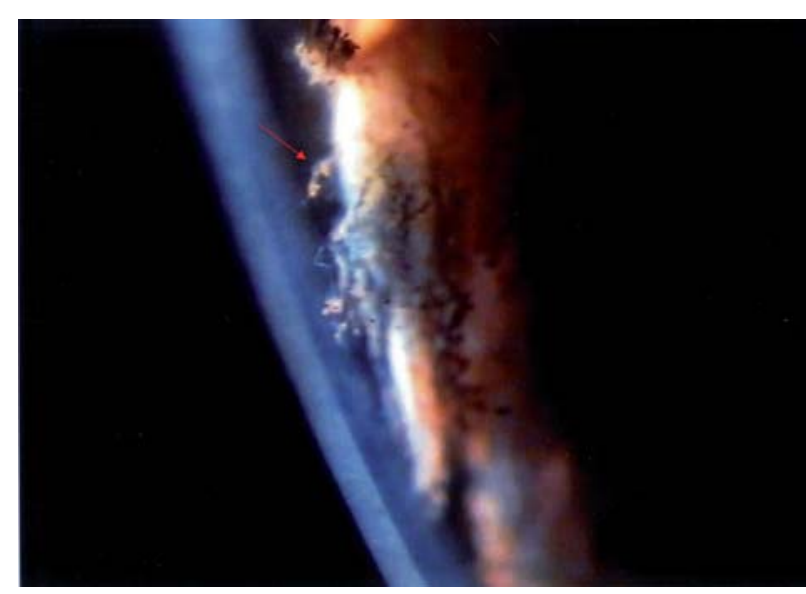

Fig. 1f: Deshilachamiento de la capa anterior del iris.

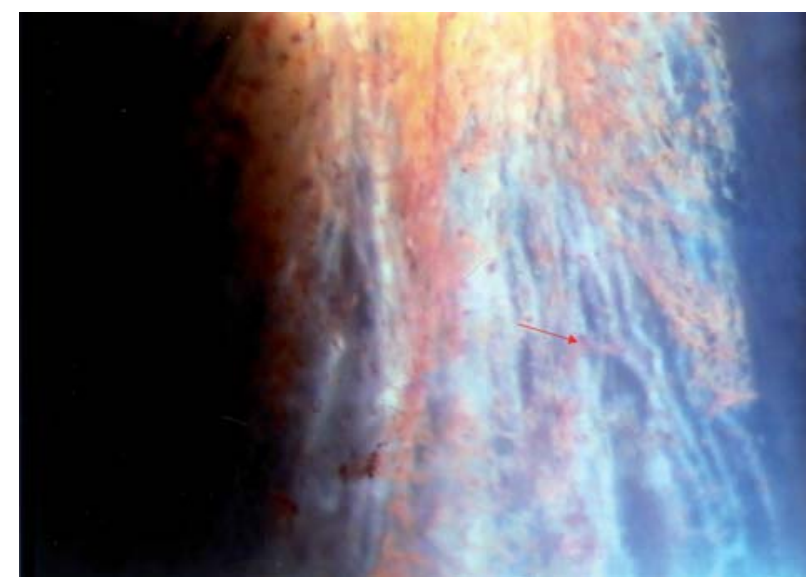

Fig. 1g: Se aprecian la disolución del estroma del iris, desestructuración completa del estroma.

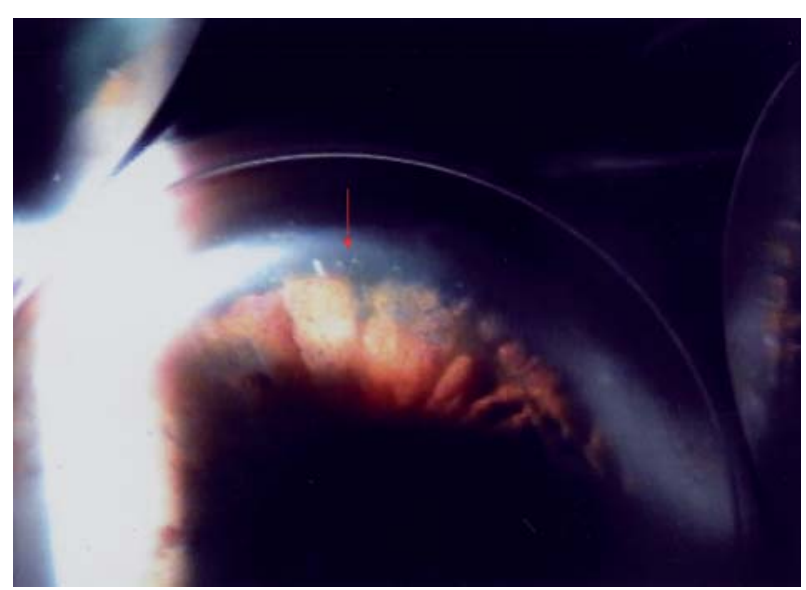

Fig. 2a: OI con mayor componente atrófico del iris.

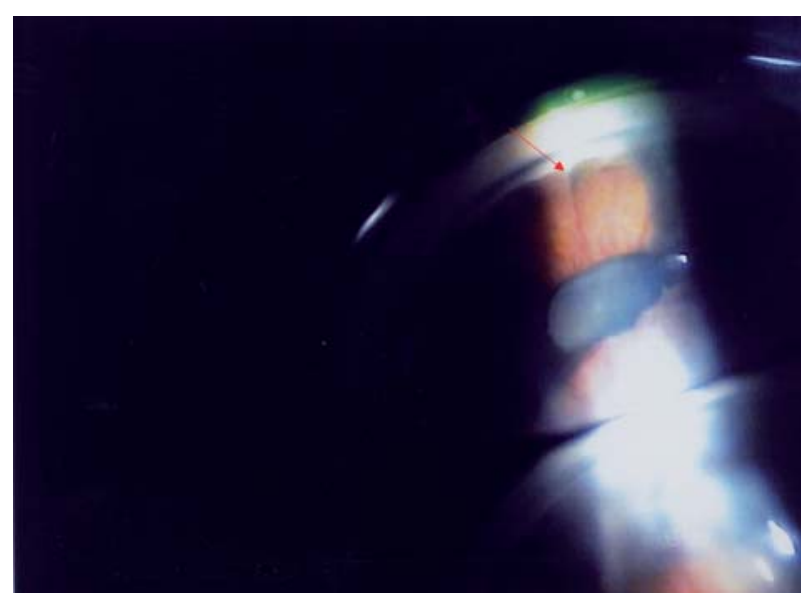

Fig. 2b: OD donde se aprecia ese iris globuloso y el estrechamiento del ángulo.

cifras de tensión ocular de $24 \mathrm{~mm} \mathrm{Hg}$. en AO, con un campo visual (Humphrey 30-2) con una reducción general de la sensibilidad (figs. 3a y 3b). Explorando el fondo de ojo encontramos lesiones hiperpigmentadas y atróficas (en «sal y pimienta») (figs. 4a y 4b).

Con el diagnóstico de presunción de sífilis congénita, aún sin leucomas propios de queratitis intersticial antigua, solicitamos pruebas serológicas para lues obteniendo resultados positivos en test treponémicos (TPHA, FTA abs) y negativos en los no treponémicos (RPR).

En este punto la paciente es evaluada por medicina interna y es cuando averiguamos que desconoce la causa real de sus conjuntivitis, aunque relata, de forma detallada: los tratamientos recibidos con 9 


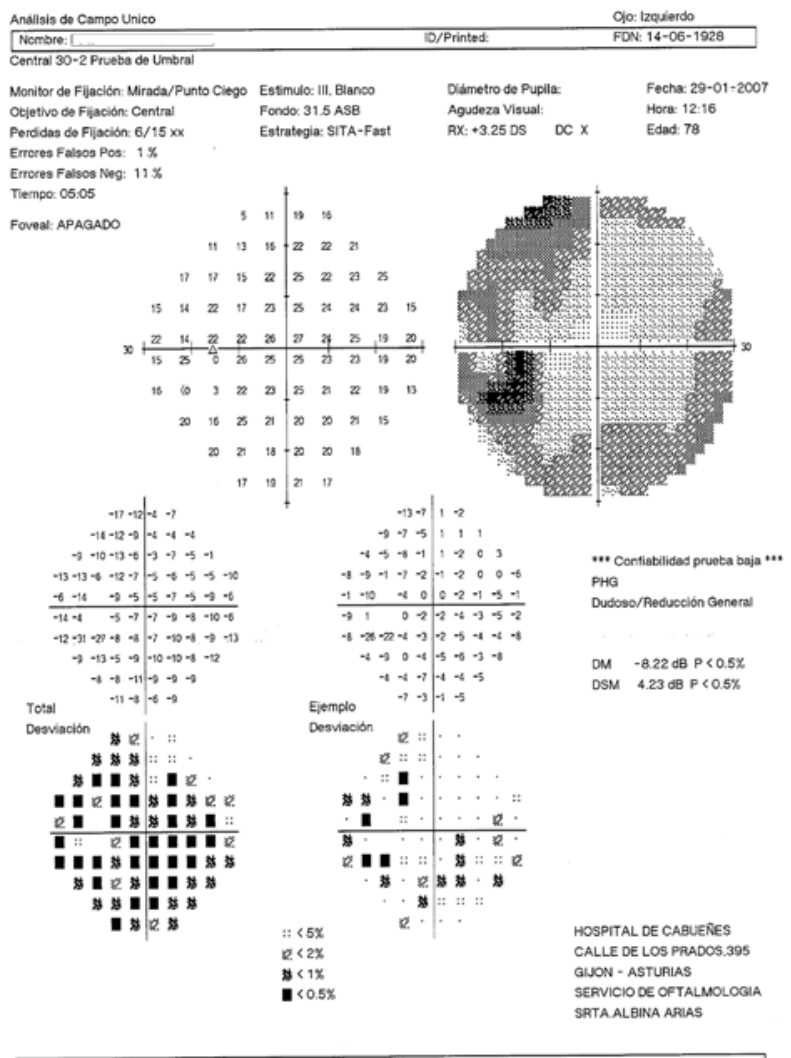

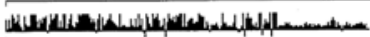

I. 2003 Carl Zeiss Mediec

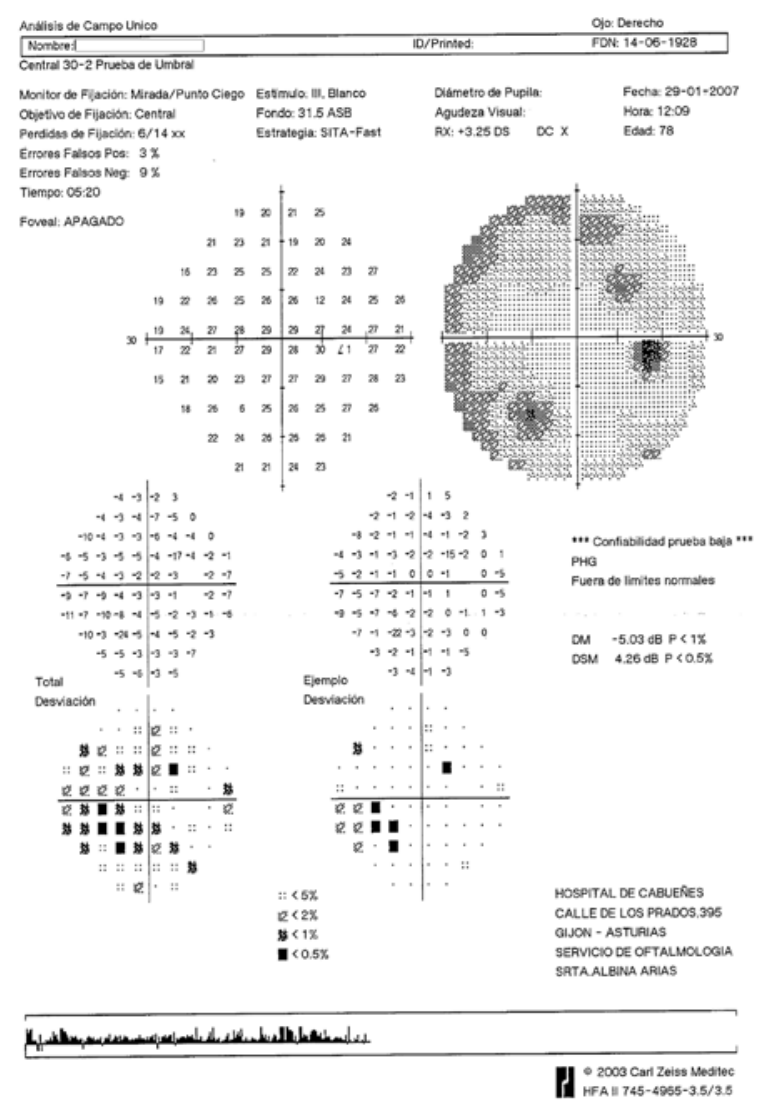

Fig. 3 a y b: Campimetría Humphrey 30-2 ambos ojos, reducción de la sensibilidad en general, patrón glaucomatoso dudoso.
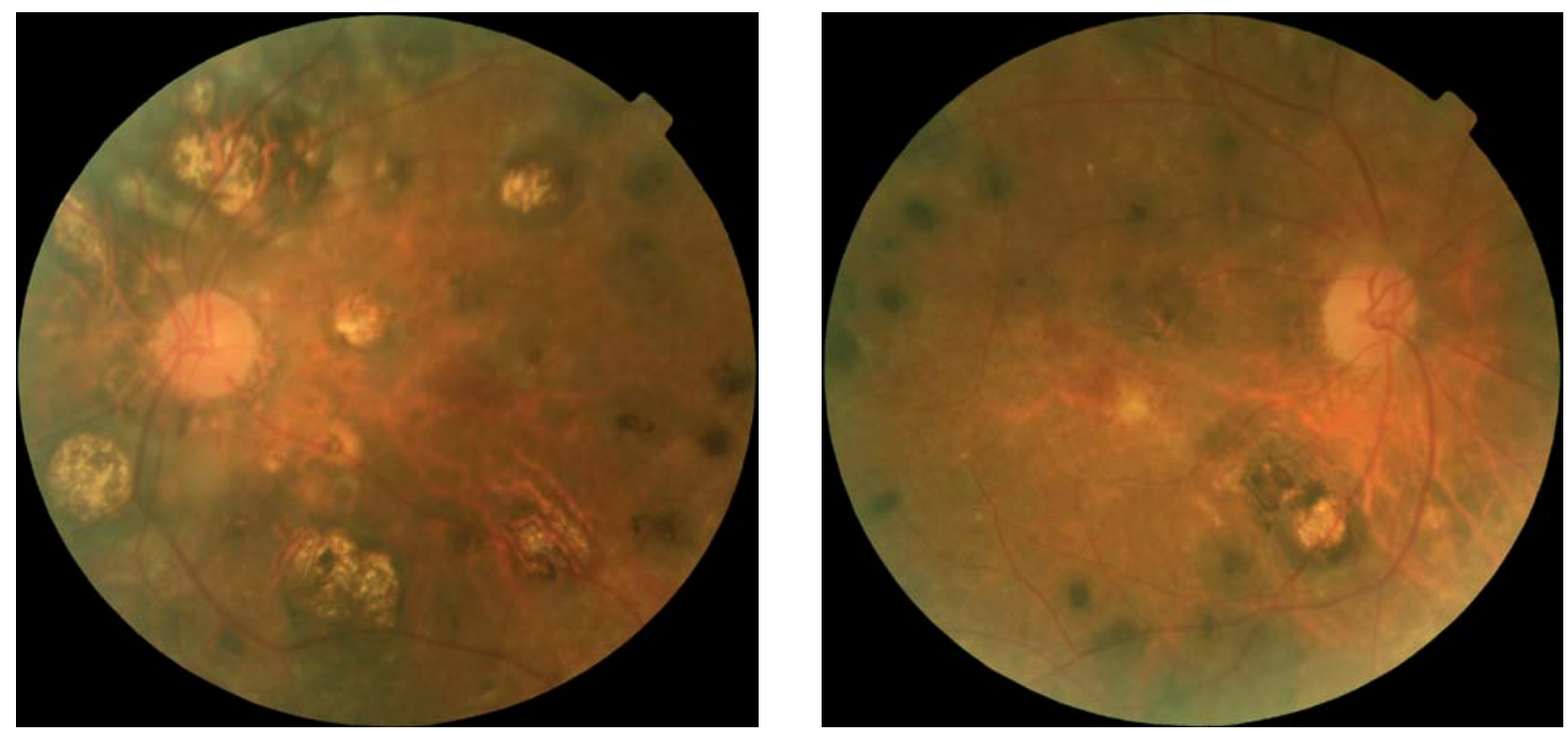

Fig. 4 a y b: Fondo de ojo, con lesiones hiperpigmentadas y otras atróficas distribuidas por polo posterior con respeto macular responsable de su buena AVC:1. 
años, en plena era preantibiótica, con neobismuto oral, que su madre «soltera» muere joven «deformada» y que en los años 60, por problemas inespecíficos articulares, le pautaron unas inyecciones semanales y mensuales (penicilina probablemente). Hallazgos de la anamnesis compatible con sífilis congénita tardía, con queratitis intersticial tratada con éxito, pues carece de leucomas. Adquirida por infección trasplacentaria: madre con artropatía severa compatible con sífilis secundaria. Y curada por resultado negativo en test RPR.

\section{DISCUSIÓN}

La iridosquisis se define como la separación de las capas estromales del iris de localización inferior y su desintegración en fibrillas que flotan en cámara anterior. Descrita inicialmente por Schmitt en 1922, el término fue propuesto por Lowenstein y Foster en 1945 que lo asociaron a cambios seniles (1-3). Desde entonces se han publicado alrededor de 50 casos asociados la mayoría a glaucoma de ángulo estrecho, a microftalmos $(1,2)$, a sífilis congénita con queratitis intersticial $(1,2,4,5)$, $\mathrm{y}$ otros $\sin$ QI, como en nuestro caso $(2,3)$.

El hecho de que sea el glaucoma de ángulo estrecho el origen más probable de la iridosquisis está por demostrar. ¿Qué es primero el glaucoma que produce isquemia en el iris provocando los cambios descritos? o ¿son los detritus del iris los que obstruyen la malla trabecular causando el glaucoma? La demostración histológica no ha llegado a esclarecerlo aún (1-3).

La asociación a la sífilis congénita inicialmente se apreció como hallazgo casual (3), aunque el aumento de publicaciones, donde la queratitis intersticial sifilítica se asocia a iridosquisis hace más plausible que el fenómeno inmunológico que ocasiona la QI sea también el origen de la Iridosquisis (1). Algunos de estos casos confirmados por tests serológicos $(1,3)$ o por una semiología clínica que sugiera el diagnóstico $(2,4,5)$.

En cuanto al serodiagnóstico se establece usando dos tipos de métodos: los ensayos no treponémicos como son el VDRL (test de laboratorio de enfermedades venéreas) o el RPR (reagina plasmática rápida) que se usan como tamizaje y para monitorizar la respuesta al tratamiento y los treponémicos: FTAAbs (Absorción de los anticuerpos treponémicos fluorescentes) y TPHA (Hemaglutinación del T.pallidum), que son pruebas confirmatorias cuya reactividad persiste a menudo para siempre como en el caso descrito.

Así estamos ante un caso interesante por múltiples datos. Los antecedentes de conjuntivitis, tratadas con pomadas con bismuto (1937), indican una resolución del cuadro ocular, dado la ausencia de leucomas invalidantes, propios de las QI de la sífilis congénita. La «buena medicina» de 1960 que con escasos medios diagnósticos, anamnesis y exploración física, y gracias a la penicilina curan una presumible artropatía sifilítica semejante a la existente en sífilis secundaria. Además nuestro hallazgo de iridosquisis bilateral, sin queratitis intersticial, con datos de coriorretinosis sifilítica no está previamente descrito, aunque en muchos casos publicados no se exploró el fondo de ojo $(3,5)$.

Destacamos pues la confirmación serológica de Sífilis Congénita, en el siglo XXI, en una mujer octogenaria que desconocía el origen de su enfermedad, probablemente debido al estigma social que rodea a la misma.

\section{BIBLIOGRAFÍA}

1. Foss AJ, Hykin G, Bejamin L. Intesrtitial keratitis and iridoschisis in congenital syphilis. J Clin Neuro-ophthalmol 1992; 12: 167-170.

2. Ruusuvara P, Setälä K, Kivelä T. Syphilitic interstitial keratitis with bilateral funnel-shaped iridocorneal adhesions: a case report. Eur J Ophthalmol 1996; 6: 6-10.

3. Salmon JF, Murray AD. The association of iridoschisis and primary angle-closure glaucoma. Eye 1992; 6: 267272.

4. Pearson PA, Amrien JM, Baldwin LB, Smith TJ. Iridoschisis associated with syphilitic interstitial keratitis. Am J Ophthalmol 1989; 107: 88-90.

5. Trevor-Roper PD. Iridoschisis. Proc Roy Soc Med 1954; 47: 721-722. 
